\title{
EDITORIAL Categorising Case Studies
}

OR is an applied subject, so illustration and discussion of real world applications are always a welcome addition to the literature. Papers of this kind tend to be given the generic label of 'case study'. In principle, case studies might be expected to provide a detailed description and commentary on a complete intervention from inception to final implementation of recommendations. In practice authors rarely achieve, or indeed aspire to this. More usually, attention is focused on a subset of the activities that constituted the intervention. This is especially likely when authors are concerned with presenting 'something new'. In these circumstances we are more likely to see accounts of the formulation and solution of quantitative models than discussion of other aspects of the intervention. This can be rather frustrating for readers interested in these 'other aspects'. Recently, in the Society's Newsletter (June 1993), Flowers (a practitioner), listed a number of features that case study papers should include to make them more useful to practitioners:

Flowers: appropriately enough for a practitioner, was concerned with effective outcomes of a reported intervention and the transferability to other situations of the methods used. A rather different list of desirable features was used by Boothroyd (1978) to rate papers in a survey of case-based literature: Boothroyd's list focus more on the quality of the intervention process in respect of problem formulation, model construction, planning for implementation and implementation of recommendations. Different people, it seems, expect different things from case-based literature.

Really, the term 'case study' is too general to be useful. Certainly it gives insufficient indication of what the objectives of the case-based paper are, and what might be gained from reading it. Thus, instead of using the generic label 'case study' it may be more helpful to employ a more detailed categorisation for case-based papers. Four categories suggest themselves:

Simple reporting Papers in this category emphasise reportage without critical comment or discussion, with the limited aim of informing readers about the general nature of an intervention. Description may be focused on the identification and measurement of modelled parameters. The use of any (quantitative) OR techniques may be described in more or less detail. The outcome, with some statement of the perceived benefits, is provided in a concluding paragraph or two. Papers in this category can provide useful examples of applications for potential clients and students who are new to OR, but their value to experienced operational researchers may be rather limited.

Problem structuring In this category the emphasis is on detailed description of the context, parties involved, relationships between parties, etc. A key concern is to describe the experience of drawing out perceptions and how a structured understanding was produced.

Applications of techniques In this category emphasis is on model formulation for well defined problems and the issue is one of developing a method for identifying optimal policies. Description begins with a definition of the problem, followed by relevant generic models and associated solution techniques, and then development of an appropriate variant of the general model. Discussion should demonstrate that the model developed was an appropriate and effective abstraction of the real system.

Methodology Papers in this category focus on the process of the intervention rather than the technical details of techniques employed. Discussion might focus on problems and issues related to one or more particular aspects of the process. For example, change management aspects of the intervention might be highlighted and examined critically.

Classifying case-based papers in terms of the above categories would go some way to giving potential readers a better idea of the scope of a 'case study'. It might also serve to encourage more papers across all categories. OR Insight certainly welcomes contributions in all four categories. However, discussion of problems encountered and their resolution is always encouraged, whatever the category of paper.

\section{Stephen Ward}

For the interested reader

$H$ Boothroyd (1978) Articulate intervention Taylor and Francis in association with the OR Society, ORASA text No 1, London, $p 14$. 16. 